\title{
Real-world management and long-term outcomes of diabetic macular oedema with good visual acuity
}

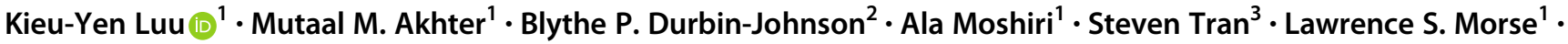 \\ Susanna S. Park $\mathbb{D}^{1} \cdot$ Glenn Yiu $\mathbb{D D}^{1}$
}

Received: 8 July 2019 / Accepted: 9 September 2019 / Published online: 28 October 2019

(c) The Author(s), under exclusive licence to The Royal College of Ophthalmologists 2019

\begin{abstract}
Purpose To evaluate the management and long-term outcomes of patients with diabetic macular oedema (DMO) and good initial visual acuity in real-world settings.

Methods We reviewed 122 eyes of 100 patients with treatment-naive DMO and initial best-corrected visual acuity (BCVA) of 20/25 or better. We assessed clinical characteristics, logMAR BCVA, central subfield thickness (CST), cumulative intravitreal injections and laser treatments at yearly intervals, and characteristics at time of initial treatment. Linear mixed effects models were used to identify predictors of visual outcomes.

Results At presentation, mean BCVA was 0.057 $\pm 0.048 \operatorname{logMAR}$ (Snellen 20/23) and mean CST was $288 \pm 57 \mu$ m. After a median follow-up of 3 years, $51 \%$ of eyes underwent treatment. More eyes underwent intravitreal injection as initial treatment $(54 \%)$, but lasers were initiated at an earlier time and at better BCVA. Final BCVA was associated with better BCVA $(P<0.001)$ and earlier timing $(P=0.017)$ at initial treatment, but not CST at first treatment $(P=0.634)$ or cumulative number of injections or lasers $(P=0.441-0.606)$.

Conclusion DMO with good initial visual acuity should be monitored closely, as delay in treatment initiation is associated with worse visual outcomes. BCVA at time of initial treatment is the strongest determinant of final visual acuity.
\end{abstract}

\section{Introduction}

Diabetic macular oedema (DMO) is a common ocular complication of diabetes resulting from disruption of the blood-retinal barrier and accumulation of fluid in the retina, which can lead to vision loss and blindness if untreated. The Wisconsin Epidemiologic Study of Diabetic Retinopathy

These authors contributed equally: Kieu-Yen Luu, Mutaal M. Akhter

Supplementary information The online version of this article (https:// doi.org/10.1038/s41433-019-0647-0) contains supplementary material, which is available to authorized users.

Glenn Yiu

gyiu@ucdavis.edu

1 Department of Ophthalmology \& Vision Sciences, University of California, Davis, Sacramento, CA, USA

2 Department of Public Health Sciences, University of California, Davis, Sacramento, CA, USA

3 Rosalind Franklin University of Medicine and Science, North Chicago, IL, USA reports that $29 \%$ of patients who have had diabetes for at least 20 years develop DMO [1]. Current treatments for DMO consists of intravitreal anti-vascular endothelial growth factor (VEGF) pharmacotherapies and focal or grid laser photocoagulation [2], as well as ancillary therapies including intravitreal corticosteroids, subthreshold micropulse laser [3-6], and pars plana vitrectomy [7, 8]. While multiple pivotal randomised prospective studies have demonstrated the efficacy of anti-VEGF agents with or without focal/grid laser as first-line treatment for DMO [9-14], most of these studies only evaluated patients with best-corrected visual acuity (BCVA) of 20/32 to 20/40 Snellen equivalent or worse. Among eyes with centreinvolving DMO and presenting BCVA of 20/25 or better in the Early Treatment Diabetic Retinopathy Study (ETDRS), $27 \%$ of eyes treated with prompt focal laser therapy lost five or more ETDRS letters compared with the $40 \%$ of eyes that were observed, supporting the role of early intervention [15]. However, this study relied on clinical examination to define "clinically significant" DMO threatening the central macula, and neither optical coherence tomography (OCT) or anti-VEGF therapy was available at the time. The more 
recent prospective Protocol V study from the Diabetic Retinopathy Clinical Research (DRCR) Retina network randomised 702 participants with centre-involving DMO with BCVA of 20/25 or better to initial management with aflibercept, laser photocoagulation, or observation only. The study found that eyes that were initially observed did not undergo significant vision loss after 2 years compared with prompt intervention with anti-VEGF [16]. However, this study did not evaluate long-term outcomes beyond 2 years, and the patients were generally healthier and more motivated-the mean haemoglobin A1c (HbA1c) was 7.6 and patients were followed closely and underwent immediate aflibercept treatment for any $>10$ letters decrease in visual acuity. To better understand the management of DMO with good initial visual acuity in real-world settings, we performed a retrospective cohort analysis of eyes with treatment-naive, centre-involving DMO and baseline BCVA of $20 / 25$ or better to assess the clinical factors associated with long-term visual and anatomic outcomes, and the impact of early versus delayed intervention.

\section{Methods}

\section{Patient selection}

We reviewed the medical records of 2262 patients diagnosed with diabetic retinopathy (ICD9 code $250 . \mathrm{XX}$ or ICD10 codes E11.311, E11.321×, E11.331×, E11.241×, E11.251X, and E11.37XX) who were seen at the University of California, Davis Health System between March 8th, 2007 to March 8th, 2018. We included only eyes with centreinvolving DMO confirmed on spectral-domain OCT imaging who had BCVA of 20/25 or better at initial diagnosis and no prior treatments, and at least 1-year of follow-up visits with SD-OCT imaging. Eyes with a history of ocular surgery or ocular comorbidities such as age-related macular degeneration, retinal vein, and artery occlusion, end-stage glaucoma, vitreomacular traction (VMT), or retinopathies unrelated to diabetes were excluded. Eyes with medically controlled glaucoma, posterior vitreous detachment, and vitreomacular adhesion without VMT were not excluded. This study was approved by the Institutional Review Board of University of California, Davis and was conducted in accordance with the tenets of the Declaration of Helsinki.

We recorded baseline demographics and clinical data including age, sex, treating provider, presence of visual symptoms, type of diabetes, and severity of diabetic retinopathy as documented by the physician at the time of diagnosis. We also recorded HbAlc levels, BCVA, lens status, cataract types and grades, central subfield thickness (CST) based on OCT imaging, and cumulative number and type of intravitreal injections or laser treatments at yearly follow-up visits. Data were collected from follow-up visits that are closest to, and within 90 days, of 12-month intervals from the initial visit, until the most recent available annual visit, up to a total of 4 years. Data beyond 4 years were not collected due to the small proportion of subjects with 5 or more years of follow-up. Snellen readings for BCVA were converted to a logarithm of the minimal angle of resolution (logMAR) scale for statistical analyses. Cataract types and grades were based on the treating physician's exam documentation, which were classified on a lens opacity scale of 0-3 for nuclear, cortical, and posterior subscapsular cataracts. Pseudophakic eyes were classified separately, and eyes that underwent cataract extraction surgery during the study period were classified as phakic prior to surgery and pseudophakic after surgery. Severity of diabetic retinopathy was classified as mild non-proliferative diabetic retinopathy (NPDR), moderate NPDR, severe NPDR, non-high-risk PDR, and high-risk PDR, based on physician documentation. CST was automatically measured as the average retinal thickness from the central $1 \mathrm{~mm}$ diameter circle cantered on the fovea, based on the ETDRS grid, using either Cirrus HD-OCT (Carl Zeiss Meditec) or Spectralis HRA + OCT instrument (Heidelberg Engineering). All OCT measurements were verified for accuracy of grid centration and retinal layer segmentation. We also recorded the length of time (in weeks) from first presentation to which the patient received the first laser treatment or intravitreal injection, the first type of laser and/or injection received, and the BCVA and CST values at the time of treatment initiation. Types of laser treatments included focal/grid laser or subthreshold micropulse laser. Types of intraocular injections included $1.25 \mathrm{mg}$ bevacizumab, $0.3 \mathrm{mg}$ ranibizumab, $2 \mathrm{mg}$ aflibercept, $2-4 \mathrm{mg}$ triamcinolone, and $0.7 \mathrm{mg}$ intravitreal dexamethasone implants.

\section{Statistical analysis}

Statistical methods were chosen to account for varying lengths of follow-up, varying providers, as well as the inclusion of patients that had both eyes qualify under the study criteria. The relationships between changes over time in VA or CST and patient baseline and disease characteristics were modelled using linear mixed effects models. These models included fixed effects for the indicated baseline/patient characteristic, year, the interaction between the indicated characteristic and year, and VA or CST at baseline, and a random effect for patient. The relationships between VA or CST at the final visit and treatment characteristics were likewise modelled using linear mixed effects models, including fixed effects for the indicated treatment characteristic, provider, lens status (phakic vs. pseudophakic), cortical, nuclear, and posterior subcapscular cataract grades, and year of last visit, and a random effect 
for patient. Analyses were conducted using R, version 3.5.3 (R Core Team, 2019), with linear mixed effects modelling conducted using the R packages lme4 (version 1.1-21) and lmerTest (version 3.1-0).

\section{Results}

\section{Demographics and baseline characteristics}

We identified 122 eyes of 100 patients with treatment-naive, centre-involving DMO and baseline BCVA of 20/25 or better. Mean age at presentation was $62.1 \pm 12.3$ years, with more men than women (63 vs. 37\%), and majority of subjects with type 2 diabetes (94\%). Most eyes were asymptomatic at the time of diagnosis (82\%), and most eyes had mild $(61 \%)$ to moderate (15\%) NPDR (Table 1). Mean baseline BCVA was $0.056 \pm 0.038$ logMAR (Snellen equivalent 20/23), which was not associated with any baseline characteristics including age, sex, symptoms at presentation, type of diabetes, diabetic retinopathy severity, or baseline CST, likely due to the homogeneity of visual acuities at baseline (Supplemental Table 1). Mean CST at presentation was $288 \pm 57 \mu \mathrm{m}$. Female sex was associated with $30.6 \mu \mathrm{m}$ lower CST compared with males $(P=0.004)$. No other baseline characteristics such as, age, symptoms, diabetes type, or diabetic retinopathy severity were associated with baseline CST (Supplemental Table 2).

\section{Management of DMO with good visual acuity}

The median follow-up duration was 3 years, with $51 \%$ of patients receiving some form of treatment during follow-up. The median time from presentation to first treatment of any type was 9.5 weeks (Fig. 1a), with mean $\log$ MAR $0.22 \pm$ 0.25 (Snellen equivalent 20/33) and mean CST of $356 \pm$ $103 \mu \mathrm{m}$ at the time of intervention. More patients underwent an intravitreal injection (54\%) than laser treatment (46\%) for initial management (Table 1), and included anti-VEGF therapy $(54.8 \%)$, followed by focal/grid laser (32.3\%), subthreshold micropulse laser (11.3\%), and only one patient receiving an intravitreal steroid (1.6\%). For eyes that received an intravitreal injection first, the median time to first intravitreal injection was 35.5 weeks (Fig. 1b), with mean $\log$ MAR $0.3 \pm 0.3$ (Snellen equivalent 20/40) and mean CST $382 \pm 107 \mu \mathrm{m}$ at the time of the first injection (Table 1). For those that underwent laser treatment first, the median time to the laser was 16.5 weeks (Fig. 1c), with mean $\log$ MAR $0.2 \pm 0.2$ (Snellen equivalent 20/31.7) and mean CST $328 \pm 81 \mu \mathrm{m}$ at the time of the first laser (Table 1). These data suggest that while intravitreal injections were the most common first intervention, laser treatments were initiated earlier and at better BCVA and CST.
Table 1 Baseline and follow-up characteristics of DMO with good visual acuity

\begin{tabular}{|c|c|}
\hline \multicolumn{2}{|l|}{ Baseline patient characteristics } \\
\hline Mean age (years) & $62.1 \pm 12.3$ \\
\hline Sex $(\%)$ (male/female) & $63 / 37$ \\
\hline Diabetes type (\%) (type 1/type 2 ) & $6 / 94$ \\
\hline \multicolumn{2}{|l|}{ Baseline eye characteristics } \\
\hline Symptoms (\%) (present/absent) & $18 / 82$ \\
\hline Laterality (\%) (right/left) & $46 / 54$ \\
\hline \multicolumn{2}{|l|}{ Diabetic retinopathy severity (\%) } \\
\hline NPDR (mild/moderate/severe) & $61 / 15 / 11$ \\
\hline PDR (non-high-risk/high-risk) & $11 / 2$ \\
\hline Mean BCVA (logMAR) & $0.057 \pm 0.048$ \\
\hline Mean CST $(\mu \mathrm{m})$ & $288 \pm 57$ \\
\hline \multicolumn{2}{|l|}{ Follow-up characteristics } \\
\hline Median follow-up (years) & 3 \\
\hline$\%$ Eyes receiving any treatment & 51 \\
\hline First treatment type (\%) (injection/laser) & $54 / 46$ \\
\hline \multicolumn{2}{|l|}{ Mean cumulative \# injections } \\
\hline Year 1 & 0.4 \\
\hline Year 2 & 1.2 \\
\hline Year 3 & 2.0 \\
\hline Year 4 & 3.1 \\
\hline \multicolumn{2}{|l|}{ Mean cumulative \# lasers } \\
\hline Year 1 & 0.3 \\
\hline Year 2 & 0.5 \\
\hline Year 3 & 0.7 \\
\hline Year 4 & 0.8 \\
\hline \multicolumn{2}{|l|}{ Median time (weeks) } \\
\hline To first treatment & 9.5 \\
\hline To first injection & 35.5 \\
\hline To first laser & 16.5 \\
\hline \multicolumn{2}{|l|}{ Mean BCVA (logMAR) } \\
\hline At first treatment & $0.22 \pm 0.25$ \\
\hline At first injection & $0.3 \pm 0.3$ \\
\hline At first laser & $0.2 \pm 0.2$ \\
\hline \multicolumn{2}{|l|}{ Mean CST $(\mu \mathrm{m})$} \\
\hline At first treatment & $356 \pm 103$ \\
\hline At first injection & $382 \pm 107$ \\
\hline At first laser & $328 \pm 81$ \\
\hline
\end{tabular}

$B C V A$ best-corrected visual acuity, CST central subfield thickness, $N P D R$ non-proliferative diabetic retinopathy, $P D R$ proliferative diabetic retinopathy

\section{Visual outcomes of DMO with good visual acuity}

During the follow-up period, BCVA decreased by $0.046 \pm$ $0.013 \log$ MAR units per year based on a linear mixed effects model (Fig. 2a), while CST measurements did not vary significantly over time across the cohort (Fig. 2b). The visual decline showed a marginal association with cortical cataract 


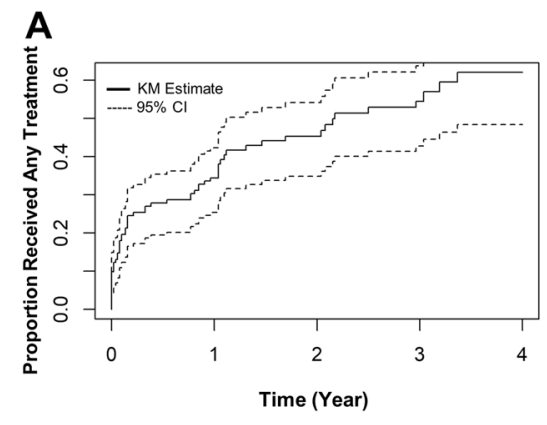

B

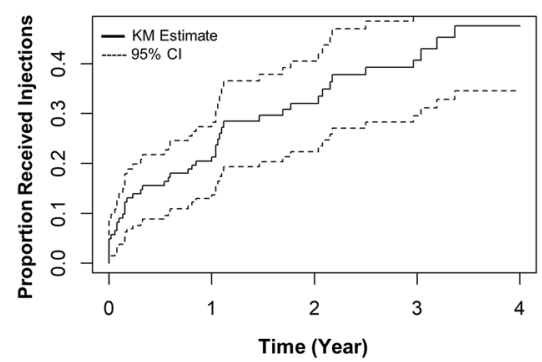

C

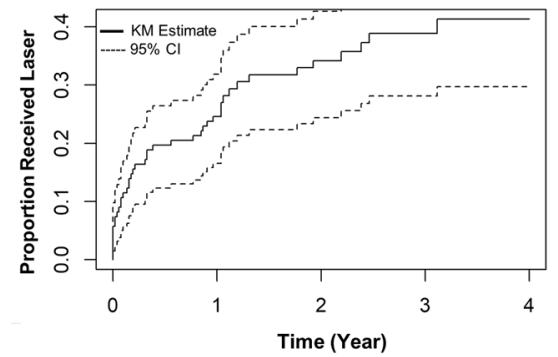

Fig. 1 Kaplan-Meier plots of the proportion of eyes with diabetic macular oedema and initial BCVA $\geq 20 / 25$ that received any treatment (a), injections (b), or laser (c). The solid line shows the KM estimate of the proportion of treated subjects, and the dashed lines show 95\% CI. CI confidence interval, ERM epiretinal membrane, KM Kaplan-Meier grade $(P=0.044)$, but not with any baseline characteristics such as age, sex, symptoms, diabetes type, retinopathy severity, or CST at presentation; or other time-varying factors such as HbA1c, nuclear sclerosis, or posterior subcapsular cataract grades (Table 2).

Next, we evaluated how management strategy impacted visual acuity outcomes. Interestingly, while the cumulative number of treatments, including either injections or lasers, did not significantly impact visual outcomes $(P=$ 0.441-0.606), there was a strong association between BCVA at the time of initial treatment and final visual acuity $(P<0.001)$. Each $0.1 \log$ MAR unit decrease in visual acuity at the time of initial treatment was associated with 0.469 (95\% CI 0.319-0.663) logMAR unit decrease in final BCVA (Table 3). The rate of visual decline was slower in eyes treated when BCVA was $20 / 25$ or better $(0.028 \pm 0.023$ $\log$ MAR units/year), compared with eyes that were not treated $(0.046 \pm 0.021 \operatorname{logMAR}$ units/year) or treated when BCVA was worse than 20/25 (0.064 \pm 0.025 logMAR units/ year) (Fig. 2c). There was also an independent association between timing of initial treatment and final visual acuity $(P=0.017)$, with each 1 -week delay in initiating therapy associated with a 0.014 (95\% CI 0.003-0.024) $\log$ MAR unit worsening in final vision (Table 3). Visual outcomes were not impacted by whether an injection or laser was chosen as initial therapy $(P=0.114)$. Among eyes that first underwent injection or laser treatment, there was a similarly strong association between better BCVA or earlier timing of intervention with better visual outcomes (Table 3). Neither the type of anti-VEGF agent used $(P=0.468)$ or modality of laser $(P=0.545)$ impacted visual outcomes within their respective subgroup analyses. Although CST at the time of first treatment did not affect final vision $(P=0.634)$, retinal thickening was also associated with worse visual outcomes in eyes that underwent laser therapy $(P=0.026)$. Together, our findings suggest that among clinical factors evaluated in this study, better BCVA at the time of initiating treatment is the strongest determinant of visual outcomes in eyes with DMO and good initial visual acuity.

\section{Discussion}

The timing for initiating treatment for eyes with DMO and good initial visual acuity has been an area of great interest for retinal specialists. While interstitial fluid and retinal thickening may damage the cellular components of the central macula responsible for high acuity visual function, patients with good visual acuity or without symptoms are often reluctant to undergo therapy based solely on anatomic findings. The ETDRS showed that $27 \%$ of eyes with centreinvolving DMO and BCVA $\geq 20 / 25$ treated with focal or grid laser lost five or more letters at 2 years compared with $40 \%$ in the observation group, supporting prompt intervention with laser therapy [15]. The DRCR Protocol I further showed that only $4 \%$ of subjects receiving antiVEGF therapy lost five or more letters at 2 years, although that study was limited to eyes with BCVA of 20/32 or worse [9]. The prospective, randomised DRCR Protocol V study suggests that eyes with DMO and initial BCVA of 20/ 25 or better may be initially observed without prompt antiVEGF therapy [16]. However, the study was limited to 2 years of follow-up, and patients enrolled in prospective clinical trials are generally healthier and more motivated to follow closely. In our study, we evaluated the real-world management of a large cohort of eyes with treatment-naive DMO and initial visual acuity $\geq 20 / 25$ over a 10 -year period and assessed their long-term visual outcomes up to 4 years of follow-up. The mean baseline HbA1c in our cohort was 8.76, compared with the mean HbA1c of 7.6 in Protocol V. The proportion of eyes that received intravitreal injections was $38 \%$ at 2 years, which is similar to the $25-34 \%$ that received aflibercept in eyes randomised to initial observation or laser in Protocol V. However, our cohort of patients underwent slow visual decline of $0.046 \log$ MAR units $(\sim 1 / 2$ Snellen line) per year, while those in protocol $\mathrm{V}$ remained largely stable near 20/20, suggesting that real-world patients with DMO and good initial visual acuity who were not closely monitored in clinical trial settings may suffer some visual loss over 3-4 years. 
A

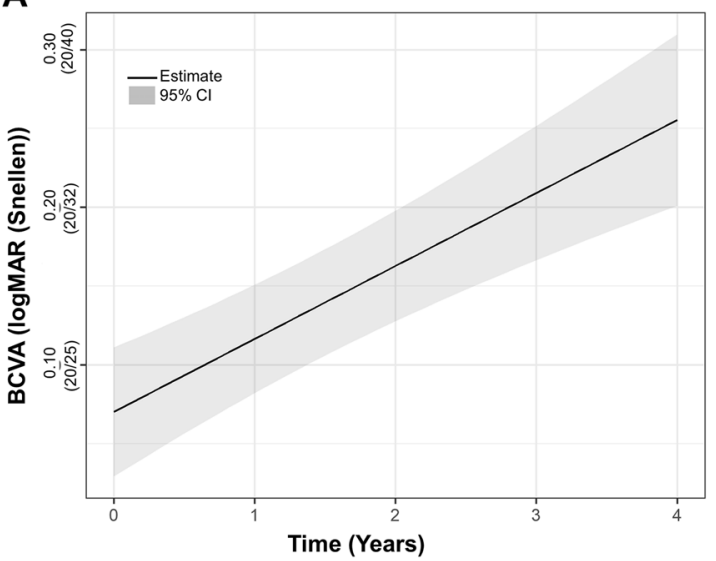

B

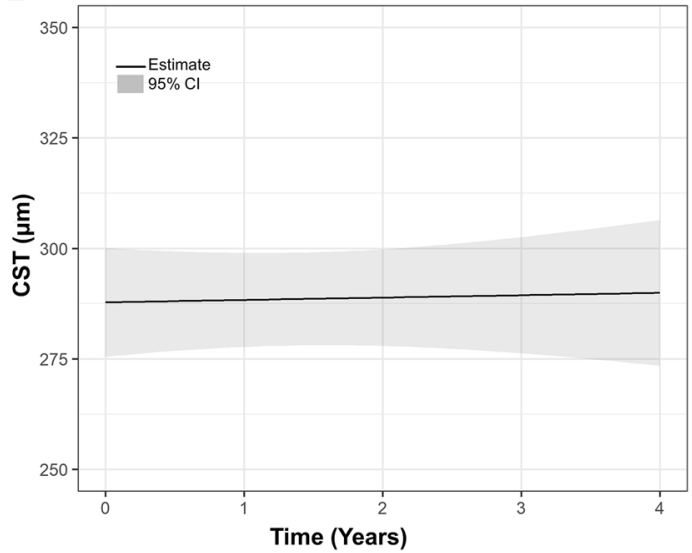

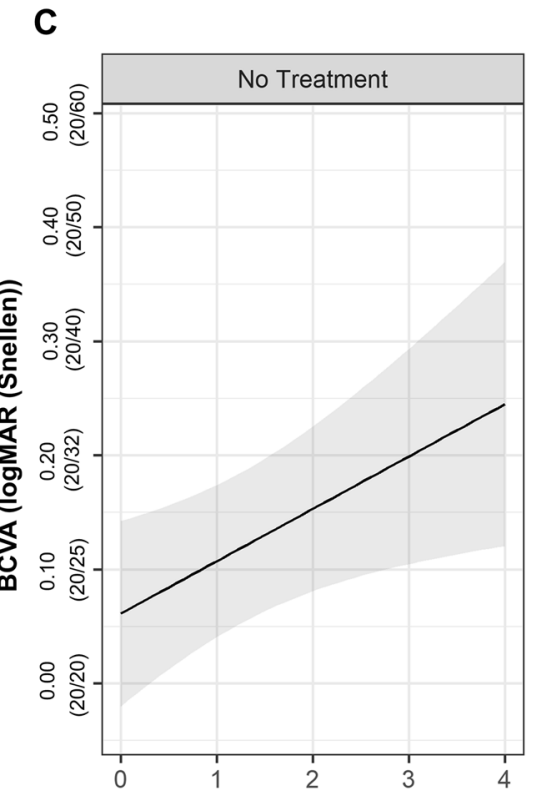
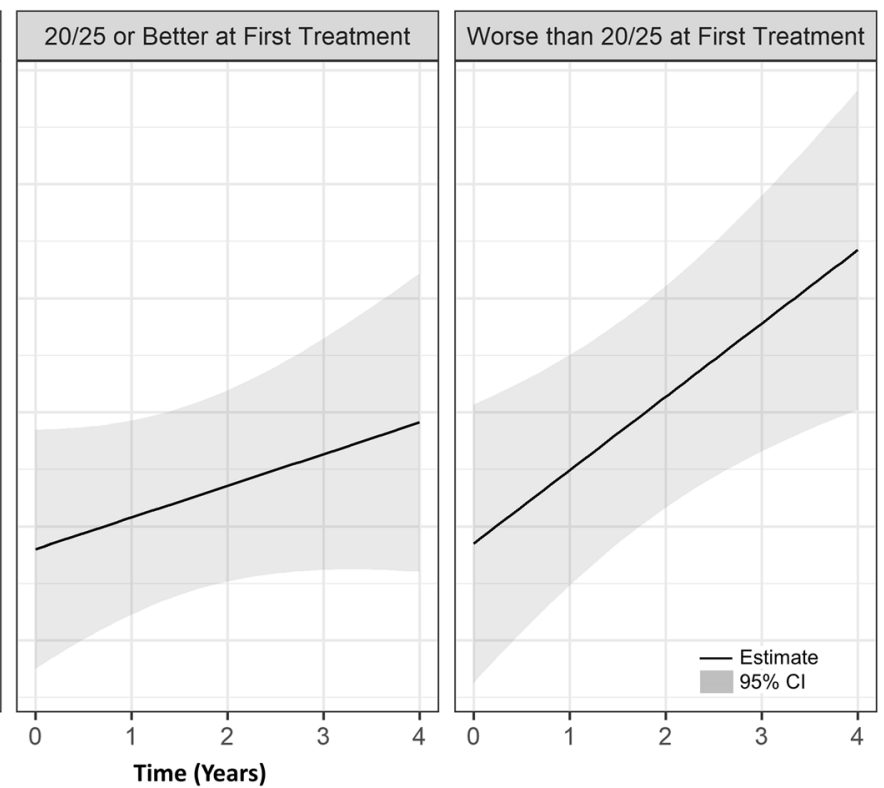

Fig. 2 Linear plots of long-term visual (a) and anatomic outcomes (b) of eyes with diabetic macular oedema and initial BCVA $\geq 20 / 25$. The solid lines on the plot shows the fit from a linear mixed effects model of BCVA or CST, with a fixed effect for year and random effects for subject and eye. The estimated rate of change in BCVA is 0.046 $\log$ MAR/year, with a $95 \%$ CI of (0.033 logMAR/year, $0.059 \log$ MAR/ year), $P<0.001$. The estimated rate of change in CST is $0.53 \mu \mathrm{m} /$ year, with a $95 \% \mathrm{CI}$ of $(-3.29 \mu \mathrm{m} /$ year, $4.39 \mu \mathrm{m} /$ year $), P=0.784$. c Linear

Unlike Protocol V where patients randomised to initial observation or laser were given prompt aflibercept treatment for a two-line decrease in visual acuity, the patients in our retrospective study were managed based on the clinician's discretion. In this setting, approximately half of our patient cohort underwent either intravitreal injection or focal/grid laser during the follow-up period, with a median time of 2.5 months to initiating some form of therapy in these eyes. Although intravitreal injections were more commonly performed as initial therapy, laser treatments tended to be initiated at an earlier time (median 16.5 vs. 35.5 weeks), and at better BCVA (mean Snellen 20/32 vs. 20/40), possibly plots of subgroup analyses of BCVA change over time in eyes that received no treatment (left), $\geq 20 / 25$ at first treatment (centre), and $<20 /$ 25 at first treatment (right). The solid lines show the fits from a linear mixed effects model of BCVA, with fixed effects for year, vision at first treatment, and their interaction, and random effects for subject and eye. The shaded grey areas for all graphs show simultaneous $95 \% \mathrm{CI}$ for the fits. BCVA best-corrected visual acuity, CI confidence interval, CST central subfield thickness

because laser treatments are perceived as better tolerated, exhibiting greater durability, and requiring less frequent follow-up visits. Also, anti-VEGF therapies may be favoured in eyes with foveal fluid, where visual acuity is more likely to be compromised, while laser photocoagulation may be better suited for non-foveal oedema. While our study did not distinguish between fluid type, amount, or location, a more robust analysis of OCT findings may provide additional insight into imaging features that could guide the management of these eyes [14].

An important finding in our study is the strong association between visual acuity at first treatment and visual 
Table 2 Clinical characteristics associated with visual outcomes in DMO with good visual acuity
Table 3 Treatment parameters associated with visual outcomes in DMO with good visual acuity

\begin{tabular}{llcc}
\hline & Category or increment & ${\text { Coefficient }(95 \% \text { CI })^{\mathrm{a}}}$ & $P$-Value \\
\hline Baseline characteristics & & & \\
Age & 1 year & $0.253(-0.181,0.69)$ & 0.256 \\
Sex & Female vs male & $0.008(-0.009,0.024)$ & 0.382 \\
Symptoms & Present vs. absent & $-0.494(-1.059,0.063)$ & 0.085 \\
DM type & Type 2 vs. type 1 & $-0.082(-0.995,0.853)$ & 0.861 \\
DR severity & 1 level & $0.063(-0.136,0.258)$ & 0.534 \\
Baseline CST & $50 \mu \mathrm{m}$ & $-0.108(-0.282,0.063)$ & 0.219 \\
Time-varying characteristics & & & \\
A1C & 1 & $-0.029(-0.162,0.104)$ & 0.671 \\
Nuclear cataract grade & 1 level & $0.265(-0.063,0.597)$ & 0.117 \\
Cortical cataract grade & 1 level & $0.361(0.013,0.71)$ & $0.044^{*}$ \\
Posterior subcapsular cataract grade & 1 level & $0.28(-0.556,1.119)$ & 0.515 \\
\hline
\end{tabular}

${ }^{*} P<0.05$, statistically significant

${ }^{a}$ For categorical variables, coefficient is the difference between categories in the rate of change over time in VA (in units of $0.1 \log \mathrm{MAR}$ ) between categories. For continuous variables, coefficient is the change in the rate of change of time in VA (in units of $0.1 \log$ MAR) for the indicted change in the continuous variable

\begin{tabular}{|c|c|c|c|}
\hline & Category or increment & Coefficient $(95 \% \mathrm{CI})^{\mathrm{a}}$ & $P$-Value \\
\hline \multicolumn{4}{|l|}{ Treatment pattern } \\
\hline Cumulative injections & One injection & $0.033(-0.085,0.155)$ & 0.606 \\
\hline Cumulative laser & One laser & $-0.2(-0.717,0.286)$ & 0.441 \\
\hline \multicolumn{4}{|l|}{ Treatment Initiation } \\
\hline $\begin{array}{l}\text { Type of first ANY } \\
\text { treatment }\end{array}$ & Injection vs. laser & $0.736(-0.093,1.589)$ & 0.114 \\
\hline \multicolumn{4}{|l|}{ Time to: } \\
\hline First treatment & 1 week & $0.014(0.003,0.024)$ & $0.017^{*}$ \\
\hline First injection & 1 week & $0.016(0.005,0.028)$ & $0.021^{*}$ \\
\hline First laser & 1 week & $0.012(0.002,0.022)$ & $0.041^{*}$ \\
\hline \multicolumn{4}{|l|}{ BCVA at: } \\
\hline First treatment & $0.1 \log$ MAR & $0.469(0.319,0.663)$ & $<0.001^{*}$ \\
\hline First injection & $0.1 \log$ MAR & $0.582(0.414,0.756)$ & $<0.001^{*}$ \\
\hline First laser & $0.1 \log$ MAR & $0.516(0.319,0.727)$ & $<0.001^{*}$ \\
\hline \multicolumn{4}{|l|}{ CST at: } \\
\hline First treatment & $50 \mu \mathrm{m}$ & $0.048(-0.123,0.229)$ & 0.634 \\
\hline First injection & $50 \mu \mathrm{m}$ & $0.037(-0.228,0.294)$ & 0.811 \\
\hline First laser & $50 \mu \mathrm{m}$ & $0.604(0.549,0.653)$ & $0.026^{*}$ \\
\hline
\end{tabular}

${ }^{*} P<0.05$, statistically significant

${ }^{a}$ For categorical variables, coefficient is the difference between categories in VA at the final visit (in units of $0.1 \log$ MAR). For continuous variables, coefficient is the change in VA at the final visit (in units of 0.1 $\log$ MAR) for the indicated change in the continuous variable outcomes-each line of BCVA increase at time of intervention was associated with $\sim 0.5$ line gain in final vision. Better visual outcomes were also associated with starting therapy earlier, but not with any baseline characteristics (demographics, symptoms, diabetes type, or severity of retinopathy), time-varying factors (HbA1c or cataract severity), total treatment burden (total injections or lasers), or CST at the time of first treatment. Hence, our results suggest that delayed treatment of DMO with good initial vision may lead to worse visual outcomes, and that initiating treatment based on BCVA may be the most important determinant of final visual acuity. These findings 
complement Protocol V findings, emphasising the need for close monitoring and prompt treatment despite the option to initially observe DMO with good initial visual acuity. Our results also support the use of visual acuity rather than anatomy as the primary determinant or threshold for initiating treatment.

Due to its retrospective nature, our study lacks the granularity of data to understand the clinical circumstances for treatment decisions, frequency or pattern of treatments, injection strategy (e.g. as-needed or treat-and-extend), switching between anti-VEGF agents, or interactions between lasers and injections. We also cannot conclude that earlier intervention would have improved the outcomes of eyes with delayed treatment. Finally, the lack of statistical difference between initiating injections vs. laser therapy or different types of injections does not imply equivalence, so we cannot conclude if starting with one treatment may be superior to another. Since prolonged anti-VEGF therapy may have secondary effects in eyes with DMO [17], care should be taken when inferring treatment decisions from retrospective analyses.

Beyond the longer duration of follow-up, our study is strengthened by the use of linear mixed effects models that adjusted for cataract grade to account for changes in lens opacity which may impact eyes with good visual acuity, and for provider identity to account for different treatment preferences of individual physicians. The study is limited by its retrospective design, which includes (1) a diversity of intervention practice patterns, (2) reliance on physician documentation of exam findings, (3) visual acuity measurements using Snellen rather than ETDRS charts, and (4) inclusion of two different commercial SD-OCT instruments for CST measurements. Nevertheless, consistent with studies in other retinal conditions where early intervention may improve visual outcomes [18], our findings supports the importance of closely monitoring patients with DMO and good baseline visual acuity, and the need to initiate therapy promptly if visual acuity declines to maximise long-term visual outcomes.

\section{Summary}

\section{What was known before}

- Anti-VEGF agents and focal/grid lasers are effective treatments for diabetic macular enema (DMO), but most prospective clinical trials evaluated only patients with best-corrected visual acuity (BCVA) of 20/32 or worse. In eyes with DMO and BCVA of $20 / 25$ or better, the DRCR Protocol V study showed that initial observation may be a reasonable strategy, if aflibercept were given for any worsening in BCVA.

\section{What this study adds}

- In real-world settings, eyes with DMO and initial BCVA greater than or equal to 20/25 undergo gradual visual decline, with $51 \%$ of eyes undergoing treatment at 3-year median follow-up. Visual outcomes were strongly associated with better BCVA at the time of treatment initiation, with each line of BCVA increase at time of intervention associated with $\sim 0.5$ line gain in final vision. This study suggests that DMO with good initial visual acuity should be monitored closely, as delay in treatment initiation may be associated with worse visual outcomes.

Funding BPD is supported by NIH UL1 TR001860. AM is supported by NIH K08 EY027463, NIH U24 EY029904. LSM received personal fees for consultancy from Genentech \& Iridex. SSP is supported by NIH 1UG1EY026876 and received grant from received grant support from Allergan and Roche Novartis. GY is supported by NIH K08 EY026101, E. Matilda Ziegler Foundation for the Blind, Barr Foundation for Retinal Research, and Macula Society, received grant support from Alcon, Clearside Biomedical, and Iridex, and personal fees for consultancy from Alimera, Allergan, Carl Zeiss Meditec, Genentech, and Iridex. No funding organisations had any role in the design or conduct of this research. The content is solely the responsibility of the authors and does not necessarily represent the official views of the funding agencies.

\section{Compliance with ethical standards}

Conflict of interest The authors declare that they have no conflict of interest.

Publisher's note Springer Nature remains neutral with regard to jurisdictional claims in published maps and institutional affiliations.

\section{References}

1. Klein R, et al. The Wisconsin epidemiologic study of diabetic retinopathy. IV. Diabetic macular edema. Ophthalmology. 1984;91:1464-74.

2. Todorich B, Yiu G, Hahn P. Current and investigational pharmacotherapeutic approaches for modulating retinal angiogenesis. Expert Rev Clin Pharm. 2014;7:375-91.

3. Lois N, et al. Diabetic macular oedema and diode subthreshold micropulse laser (DIAMONDS): study protocol for a randomised controlled trial. Trials. 2019;20:122.

4. Chen G, et al. Subthreshold micropulse diode laser versus conventional laser photocoagulation for diabetic macular edema: a metaanalysis of randomized controlled trials. Retina. 2016;36:2059-65.

5. Moisseiev E, et al. Subthreshold micropulse laser reduces antiVEGF injection burden in patients with diabetic macular edema. Eur J Ophthalmol. 2018;28:68-73.

6. Vujosevic S, et al. Subthreshold micropulse yellow laser versus subthreshold micropulse infrared laser in center-involving diabetic macular edema: morphologic and functional safety. Retina. 2015;35:1594-603.

7. Hagenau F, et al. Vitrectomy for diabetic macular edema: optical coherence tomography criteria and pathology of the vitreomacular interface. Am J Ophthalmol. 2019;200:34-46. 
8. Hu XY, et al. Efficacy and safety of vitrectomy with internal limiting membrane peeling for diabetic macular edema: a Metaanalysis. Int J Ophthalmol. 2018;11:1848-55.

9. Diabetic Retinopathy Clinical Research N. et al. Randomized trial evaluating ranibizumab plus prompt or deferred laser or triamcinolone plus prompt laser for diabetic macular edema. Ophthalmology. 2010;117:1064-77 e1035.

10. Nguyen QD, et al. Ranibizumab for diabetic macular edema: results from 2 phase III randomized trials: RISE and RIDE. Ophthalmology. 2012;119:789-801.

11. Heier JS, et al. Intravitreal aflibercept for diabetic macular edema: 148-week results from the VISTA and VIVID studies. Ophthalmology. 2016;123:2376-85.

12. Korobelnik JF, et al. Intravitreal aflibercept for diabetic macular edema. Ophthalmology. 2014;121:2247-54.

13. Diabetic Retinopathy Clinical Research N. et al. Aflibercept, bevacizumab, or ranibizumab for diabetic macular edema. New Engl J Med. 2015;372:1193-203.
14. Wells JA, et al. Aflibercept, bevacizumab, or ranibizumab for diabetic macular edema: two-year results from a comparative effectiveness randomized clinical trial. Ophthalmology. 2016;123:1351-9.

15. Photocoagulation for diabetic macular edema. Early Treatment Diabetic Retinopathy Study report number 1. Early Treatment Diabetic Retinopathy Study research group. Arch Ophthalmol. 1985;103:1796-806.

16. Baker CW, et al. Effect of initial management with aflibercept vs laser photocoagulation vs observation on vision loss among patients with diabetic macular edema involving the center of the macula and good visual acuity: a randomized clinical trial. JAMA. 2019;321:1880-94.

17. Yiu G, et al. Effect of anti-vascular endothelial growth factor therapy on choroidal thickness in diabetic macular edema. Am J Ophthalmol. 2014;158:745-51 e742.

18. Moisseiev E, et al. 25-gauge vitrectomy with epiretinal membrane and internal limiting membrane peeling in eyes with very good visual acuity. Curr eye Res. 2016;41:1387-92. 